\title{
Une ville en politique Augsbourg à la fin du Moyen Âge
}

\section{Dominique Adrian}

\section{OpenEdition}

\section{Journals}

\section{Édition électronique}

URL : http://journals.openedition.org/ifha/175

DOI : 10.4000/ifha. 175

ISSN : 2198-8943

\section{Éditeur}

IFRA - Institut franco-allemand (sciences historiques et sociales)

\section{Édition imprimée}

Date de publication : 6 février 2011

Pagination : 126-132

ISSN : 2190-0078

\section{Référence électronique}

Dominique Adrian, « Une ville en politique Augsbourg à la fin du Moyen Âge », Revue de l'IFHA [En ligne], 3 | 2011, mis en ligne le 10 février 2012, consulté le 01 mai 2019. URL : http:// journals.openedition.org/ifha/175; DOI : 10.4000/ifha.175

Ce document a été généré automatiquement le 1 mai 2019.

(CIFHA 


\title{
Une ville en politique Augsbourg à la fin du Moyen Âge
}

\author{
Dominique Adrian
}

\section{NOTE DE L'ÉDITEUR}

Dominique Adrian a soutenu sa thèse de doctorat sous la direction de Jean-Marie Moeglin en décembre 2009, à l'université Paris Est-Créteil-Val-de-Marne. Elle portait le titre :

" Augsbourg à la fin du Moyen Âge : la politique et l'espace ».

Dans les deux derniers siècles du Moyen Âge, Augsbourg n'est pas encore la métropole économique de premier plan qu'elle sera à la Renaissance, mais elle est déjà l'une des principales villes d'Allemagne du Sud, au sein d'un réseau constitué majoritairement de villes d'Empire comme elle, constamment en relation et réunies par une culture largement partagée. Augsbourg, avec ses quelque quinze à vingt mille habitants vers 1500 et ses compagnies commerciales en plein développement, joue dans ce réseau un rôle essentiel à la hauteur de celui de Nuremberg ou d'Ulm.

Comme à Ulm, comme dans la plupart des villes de l'aire souabe et franconienne, et contrairement à Nuremberg, le fait marquant dans la vie politique augsbourgeoise à la fin du Moyen Âge est la mise en place d'un régime politique dominé par les représentants des métiers, succédant à un gouvernement exclusif des patriciens. Le processus augsbourgeois se singularise pourtant par deux éléments : d'une part, il faut attendre 1368 pour que les métiers accèdent au pouvoir; d'autre part, l'arrivée massive des métiers au pouvoir est réalisée en une seule fois, sans violence, et n'est jamais réellement remise en cause avant le XVIe siècle. C'est donc un régime presque inchangé qui est en place de 1368 à son abolition, par décision de Charles Quint, en 1548: si des conflits ont bien lieu, notamment au cours d'une période agitée entre 1456 et 1478, si des évolutions souvent souterraines l'adaptent au cours de sa longue existence, la structure 
institutionnelle montre ainsi sa capacité à amortir et rendre compte des évolutions sociales et politiques.

Pour connaitre ces cent quatre-vingts ans de vie politique augsbourgeoise, l'historien dispose avant tout du vaste ensemble constitué par les archives du Conseil, encore aujourd'hui conservées aux archives municipales d'Augsbourg. Cet ensemble n'est pas qu'une riche documentation qui éclaire largement la vie des institutions augsbourgeoises et celle des hommes qui les peuplent : leurs contours, leur évolution dessinent aussi, à travers l'extension du champ de l'écrit, une histoire de l'ancrage du champ politique - à travers les institutions, mais aussi la culture politique ou l'investissement humain qui le fait vivre - dans la société urbaine. Le régime des métiers hérite des patriciens des compétences et des habitudes de recours à l'écrit : pour la perception de l'impôt, pour l'enregistrement des nouveaux bourgeois, pour le suivi des finances urbaines. Mais ce dernier domaine est précisément celui où le changement de pratiques est le plus frappant. Il ne reste presque rien des comptabilités patriciennes, sans doute jamais destinées à la conservation; dès la fin de 1368, les nouveaux maîtres du Conseil commencent une longue série de registres comptables en tirant parti du travail accompli par leurs prédécesseurs au long de l'année écoulée : ce n'est donc pas une différence de compétence, puisqu'ils ne font que reproduire des habitudes d'enregistrement et de hiérarchie comptable, c'est bien l'idée tacite que la réalisation d'une comptabilité vérifiable, et donc écrite, est l'indispensable corollaire du changement institutionnel. Il en va un peu de même pour les registres du Conseil, même si l'évolution est beaucoup plus tardive : il faut attendre 1390 pour relever la trace d'une volonté de conservation cohérente des décisions du Conseil en un registre ad hoc, et ce n'est qu'au cours des quatre premières décennies $d u$ XVe siècle que se met en place progressivement un modèle pérenne pour préserver la trace de l'activité législative du Conseil, au moins autant d'ailleurs à des fins de légitimation que pour en faciliter l'usage, qui est particulièrement mal attesté.

$\mathrm{Du}$ point de vue institutionnel, ces sources permettent de voir vivre les institutions définies assez précisément par des chartes de 1368, complétées au fil des besoins de la pratique. Le fondement indispensable du système est constitué par dix-sept Zünfte, associations à base professionnelle au sein desquelles se déroulent des élections qui désignent leurs dirigeants, lesquels constituent de droit les différents conseils; elles ont vocation à accueillir en leur sein la totalité de la population augsbourgeoise hors patriciens et clercs, le séjour prolongé des étrangers sans droit de bourgeoisie étant constamment combattu et l'obtention de la bourgeoise étant conditionnée par l'entrée dans une Zunft. Ces structures peuvent n'être que des coquilles vides réduites à leur fonction électorale, comme c'est longtemps le cas chez les marchands, mais deviennent aussi le lieu d'une vie sociale intense, renforcée au cours du XVe siècle par l'acquisition, par la plupart des Zünfte, d'une maison qui leur sert de lieu de réunion et de sociabilité. Les registres que tiennent ces métiers, dont seuls quelques exemples isolés subsistent aujourd'hui, constituent une occasion rare de s'intéresser à la culture des métiers artisanaux, qui ne sont pas les derniers à entreprendre la réalisation de registres où leurs affaires internes voisinent volontiers avec les grands textes fondateurs du régime des métiers.

Les élus de chaque métier, complétés par un petit nombre de patriciens, forment ensuite le Conseil, connu sous trois instances. Outre le Conseil des anciens, qui ne constitue qu'un supplétif formé de responsables expérimentés en marge du jeu politique, le Petit Conseil - 
où figure le maître de chaque Zunft et, pour les plus importantes, un autre élu - et le Grand Conseil - formé de la réunion des Douze de chaque Zunft - ne sont pas tant des conseils séparés que des formations différentes de l'entité unique qu'est le Conseil : si leur composition est différente, leurs attributions sont sensiblement identiques et le choix de convoquer l'un ou l'autre n'est affaire que d'opportunité politique et de nécessité pratique. Le Grand Conseil, trop nombreux pour être réuni trop souvent, l'est tout de même plusieurs fois par an, et chaque fois que les élites politiques jugent nécessaire d'obtenir un large assentiment à leurs projets, non sans courir le risque d'une contestation parfois efficace.

L'existence de ces différentes instances ne doit pas être pensée sous forme d'opposition, entre un Petit Conseil détenteur de la réalité du pouvoir et un Grand Conseil qui ne serait qu'une façade ou, au mieux, un forum des mécontentements populaires. La forme de la réunion du Conseil n'a pas d'incidence sur la valeur juridique des décisions prises ; le Petit Conseil a pour lui la fréquence de ses réunions et une plus grande professionnalisation de ses membres, mais le Grand Conseil dispose - au moins pendant une grande partie du XVe siècle - d'une puissance légitimante qui en rend la réunion souvent indispensable et lui donne une réelle capacité d'infléchir la position préparée par le Petit Conseil. Il en va un peu de même d'une autre institution, créée progressivement à partir de 1400 : les Treize, composés des principaux magistrats municipaux et de conseillers élus, fonctionnent largement comme une commission permanente du Petit Conseil, beaucoup plus que comme un pouvoir de l'ombre.

$\mathrm{Au}$ sommet du système se trouve un certain nombre de magistrats municipaux, principalement les deux maires et les trois Baumeister (à l'origine responsables des travaux municipaux, chargés de la totalité de l'administration financière) qui, mieux encore que les conseils, montrent l'importance de la parité dans le système augsbourgeois : un des deux maires est patricien, de même qu'un des trois Baumeister. Reste à savoir dans quelle mesure les familles patriciennes, en constant déclin démographique, peuvent encore peser dans la vie politique: quelques attestations tendent à montrer que les élus patriciens des dernières décennies du régime des métiers ne sont que des figurants sur la scène politique, d'où ils tirent une légitimation de leur statut particulier plus qu'un réel pouvoir.

Ces équilibres sont remis en question à partir des années 1450, à la suite de campagnes militaires qui ont ébranlé les finances de la ville. En 1456 puis en 1466, deux commissions examinent les finances de la ville pour tenter d'alléger les charges pesant sur elle et, par ricochet, sur les habitants : la première se contente d'un toilettage prudent, la seconde, à la composition sociale élargie, s'en prend plus directement aux intérêts des élites urbaines, et ses conclusions figurent dans de nombreux livres des Zünfte. Les troubles culminent pourtant dans la décennie suivante, pendant les quelques années de "règne » du maire Ulrich Schwarz, membre de la commission de 1466 et partisan d'un retour à l'esprit des institutions représentatives de 1368. Ce bref moment de radicalité s'achève en 1478 par l'exécution de Schwarz. Les décennies suivantes sont un moment de consolidation oligarchique du système, qui se caractérise par un maintien formel des institutions existantes, mais aussi par de grandes évolutions. La réélection annuelle des membres du Conseil, voire des magistrats, devient la norme, si bien que le rôle du Grand Conseil s'en trouve amoindri. Dans le domaine documentaire, ce n'est certainement pas un hasard si c'est à cette période que la qualité des séries de registres municipaux, principalement les registres comptables et législatifs, se dégrade de façon frappante: 
l'idée de transparence qui avait présidé à leur mise au point, très importante au moment de la mise en place du régime mais aussi auprès des partisans des réformes de 1466, devient dès lors secondaire, sinon gênante.

Outre les perspectives documentaires et institutionnelles suivies jusqu'ici, la politique peut aussi être abordée sous l'angle de ceux qui la font. À Augsbourg à la fin du Moyen Âge, ce n'est pas faire preuve d'idéalisme que de remarquer la très large implication du corps civique dans la vie politique. Non seulement la participation aux élections annuelles est une obligation - sans qu'il soit possible d'évaluer à quel point elle est respectée -, mais encore la nécessité de représenter sa Zunft au Grand Conseil met en contact des bourgeois de tout niveau social avec le gouvernement de la ville: au début du XVe siècle, un cinquième des membres de la Zunft des forgerons est amené à y siéger au moins un an.

La sociologie du milieu politique est tout d'abord marquée par la dualité entre patriciens et autres bourgeois. Cette dualité n'est pas une ligne de fracture politique, tant les patriciens sont unis par d'innombrables liens au reste des élites urbaines, même si leur distinction juridique est parfois sujet de moqueries de la part de ceux qui cherchent à restreindre la part des élites dans le monde politique, à commencer par Schwarz. Leur relation à la politique n'en est pas moins différente, ne serait-ce que du fait de leur démographie déclinante; l'accès aux fonctions les plus élevées est beaucoup moins le fruit d'une vocation individuelle qu'une nécessité, pour maintenir le privilège qui les distingue. Encore les différentes familles ont-elles un rapport différent avec la politique : ce n'est certainement pas un hasard si la famille la plus présente tout au long du XVe siècle est de celles qui n'ont pas su ou voulu confirmer leur position éminente par des activités commerciales développées, d'où une fortune modeste.

Les membres des métiers, eux, suivent en général un cursus honorum plus ou moins lent, qui passe par la présence dans la direction collective du métier, puis au poste de maître et éventuellement aux différentes magistratures municipales. Rien n'empêche des carrières plus rapides et rien ne garantit contre les accidents de parcours, sans doute plus fréquents qu'on ne le pense en général, en fonction de luttes de pouvoir internes à chaque métier, mais aussi de réelles divergences politiques, $a$ fortiori dans des métiers où des élites fortunées s'opposent à une masse d'artisans. Les métiers les plus authentiquement artisanaux, dont le rôle politique réel est certainement très faible, sont souvent ceux où les traces d'une culture politique propre sont les plus nettes, à travers les livres qu'ils réalisent, mais aussi à travers une réglementation qui favorise le vote majoritaire et le rôle de l'assemblée générale du métier, au détriment de sa direction élue. Il n'en est pas moins évident que ces élus émanent en général des élites du métier, mais il n'est pas certain que cela corresponde tellement à une confiscation du pouvoir par celles-ci : dès lors qu'il s'agit de faire porter la voix du métier parmi les instances supérieures, il peut être pertinent d'élire celui qui maîtrise le mieux les codes et les pratiques du monde politique, plutôt que celui qui partage le mieux les conditions de vie de tous.

La largeur du corps civique augsbourgeois et la large composition d'un Grand Conseil à l'influence réelle font du régime augsbourgeois l'un des plus ouverts socialement pendant l'essentiel du XVe siècle. Les choses changent à la fin du siècle après l'épisode Schwarz, qui cristallise des tendances à la fermeture déjà à l'œuvre depuis plusieurs décennies, face à la contestation latente d'une partie des métiers ; du point de vue du personnel politique, cela se traduit notamment par la généralisation de la réélection presque systématique des responsables politiques, y compris les simples membres des directions collectives des 
Zünfte artisanales, ainsi intégrées au monde politique et solidaires du Conseil avant d'être solidaires de leurs collègues.

En marge du monde politique, le monde des employés du Conseil est largement dominé par la figure des secrétaires municipaux, qui forment la direction de la chancellerie municipale et sont souvent impliqués dans les grandes orientations politiques. On attend d'eux un rôle de conseil au service du gouvernement municipal, qui peut leur donner un rôle déterminant et parfois encombrant: l'un d'eux, Heinrich Erlbach, est démis de ses fonctions en 1459 pour avoir trop ardemment soutenu les mesures réformatrices souhaitées par les métiers mécontents de l'état des finances municipales.

Ce monde politique s'incarne dans l'espace de la ville, qui ne possède pas de territoire significatif, et se trouve donc limité par le tracé des murs construits à la fin du XIVe siècle. Le gouvernement municipal ne module jamais son action en fonction de critères territoriaux (noyau historique contre faubourgs...), mais il n'en doit pas moins maîtriser l'espace pour la rendre efficace : l'adaptation des secteurs qui servent au prélèvement de l'impôt à l'évolution de l'organisme urbain en est un exemple parlant. L'hôtel de ville et la zone qui l'entoure sont au cœur de l'action du Conseil, mais la question du contrôle exercé réellement sur l'ensemble de la ville est complexe. Le XVe siècle voit l'extension de la surveillance municipale de l'ensemble de l'espace, à partir des portes, dans une perspective de renforcement du contrôle social; mais on constate qu'une bonne partie de la législation urbanistique n'entend être qu'un instrument d'arbitrage à l'usage de l'élite sociale et n'est vraiment appliquée que dans les quartiers qu'habite cette dernière.

Outre l'hôtel de ville, la présence du pouvoir dans la ville se manifeste par les maisons que les Zünfte acquièrent progressivement à partir des années 1380. Ces maisons sont multifonctionnelles : elles peuvent être un lieu de l'activité professionnelle des membres, mais aussi un lieu de réunion ou de sociabilité quotidienne. La diffusion imitative d'une norme comme celle de la possession d'une maison pose indirectement la question des lieux utilisés par les Zünfte avant ces acquisitions. Par leur implantation, les métiers se coulent ainsi dans la logique centralisatrice du pouvoir et de la richesse - à l'instar des patriciens, dont l'organisation collective était restée longtemps beaucoup plus faible -, et ce n'est sans doute pas un hasard si c'est à la même époque que le régime politique voit ses tendances à la centralisation renforcées. 